\title{
INCONTINENCIA URINARIA TRAS PROSTATECTOMÍA RADICAL: EVOLUCIÓN HISTÓRICA DE LA TÉCNICA QUIRÚRGICA Y ESTADO ACTUAL DEL RESULTADO FUNCIONAL
}

\author{
Francisco José Delgado Oliva, Juan Pablo Caballero Romeo, Diego García Serrado, Luis Prieto \\ Chaparro, Carlos Carro Rubias, Silvia Chillón Sempere, Lucas De Paz Cruz y Ernesto De Nova \\ Sánchez.
}

Servicio de Urología. Hospital General Universitario Elche. Elche. Alicante. España.

\begin{abstract}
Resumen.- OBJETIVO: La prostatectomía radical (PR) es el tratamiento de elección en los pacientes con cáncer de próstata organoconfinado y una esperanza de vida mayor de 10 años. La cirugía radical de la próstata debe incluir unos buenos resultados oncológicos y funcionales, con un mantenimiento precoz de la continencia y potencia sexual postquirúrgicas. En manos expertas la PR ofrece muy bajas tasas de incontinencia urinaria postprostatectomia.
\end{abstract}

Analizar la evolución y los resultados funcionales en cuanto a la incontinencia urinaria (IU) tras PR que tenemos en nuestro centro en los últimos diez años.

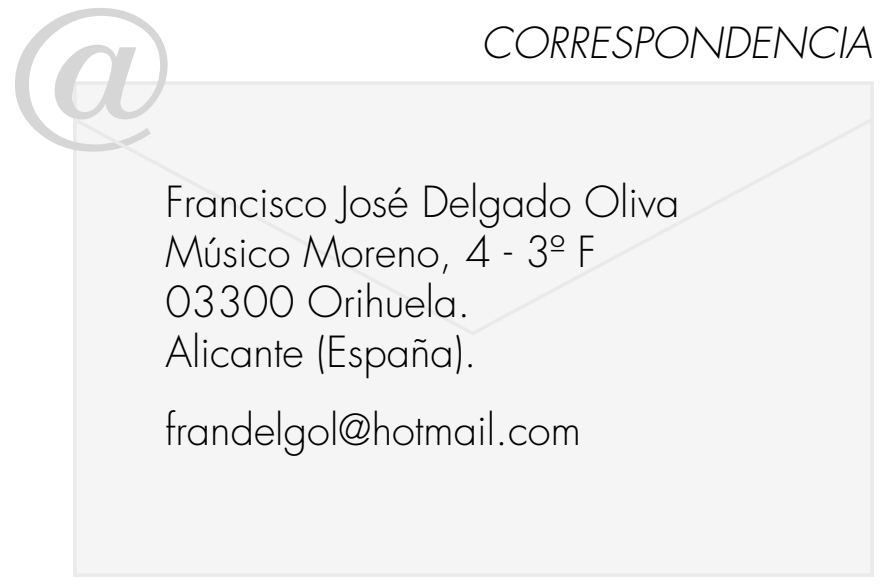

MÉTODOS: Hemos realizado un estudio retrospectivo de los 137 pacientes sometidos a prostatectomía radical (retropúbica y laparoscópica) en el Hospital General Universitario de Elche, en el período comprendido entre 1998 y 2008. Se analizaron dos grupos de pacientes, los intervenidos de 1998-2003 y aquellos que se operaron del 2004-2008, ambos inclusive. En ambos grupos se determinó el porcentaje de IU de esfuerzo tras $P R$, mediante el número de compresas utilizadas al día por cada paciente, al mes, 3 meses, 6 meses y al año de la cirugía. También se analizaron el número de estenosis de la anastomosis uretrovesical en cada grupo y cómo se resolvieron.

RESULTADOS: De los 137 pacientes iniciales, se excluyeron 15 de ellos por tratarse de estadios CT3, resultando el total en 122 prostatectomías con estadio < cT2c.La edad media de los pacientes fue de 67 años (50-74). La media del PSA preoperatorio fue de 8,1 $\mathrm{ng} / \mathrm{ml}$ (4,5-25). El Gleason en la biopsia fue < de 6 en el $70 \%$ (85/122) y de 7-8 en el $30 \%$ (37/122). En el periodo comprendido entre 1998-2003 se intervinieron 49 pacientes y se obtuvieron los siguientes resultados: Continentes de inicio: 28,5\% (14/49), continentes al mes: 6, 1 \% (3/49), continentes a los 3 meses: 12,2 $\%(6 / 49)$, continentes a los 6 meses: 8, $1 \%(4 / 49)$, continentes al año: 22,4\% (1 1/49). Quedaron con IU leve el 20,4\% (10/49) y con IU moderada/severa el $2 \%(1 / 49)$. En este periodo el $78 \%$ de los pacientes resultaron continentes y el $22 \%$ incontinentes.

En el periodo de 2004-2008 se realizaron 73 prostatectomías radicales con las siguientes tasas de continencia: Continentes de inicio: $44 \%$ (32/73), continentes al mes: 0 pacientes, continentes a los 3 meses: $6,8 \%$ (5/73), continentes a los 6 meses: 12,3\% (9/73), continentes al año: 24,6\% (18/73). Quedaron con IU leve el $8,2 \%(6 / 73)$ y con IU moderada/severa el $4 \%$ 
(3/73). Resultaron continentes un total del $88 \%$ de los pacientes e incontinentes el $12 \%$.

CONCLUSIONES: La incontinencia urinaria es un trastorno poco frecuente en los pacientes sometidos a PR por cáncer de próstata. Las tasas de continencia han ido mejorando en los últimos años con la mejora de la técnica quirúrgica y con la experiencia del urológo. Por este motivo, los dispositivos antiincontinencia tras PR son necesarios en contadas ocasiones y su uso está sobredimensionado.

Palabras clave: Cáncer próstata. Prostatectomía. Incontinencia urinaria.

Summary.- OBJECTIVES: In patients with localized prostate cancer and life expectancy longer than 10 years, radical prostatectomy (RP) remains the Gold Standard. Radical surgery must achieve good oncological and functional outcomes with early continence and potency. In expert hands RP offers very low post-prostatectomy urinary incontinence rates.

To analyze functional outcomes regarding urinary incontinence (UI) after RP in our centre in the last ten years.

METHODS: We have performed a retrospective study of the 137 patients who had a radical prostatectomy (open retropubic or laparoscopic) on the General University Hospital of Elche from 1998 to 2008. Two patient groups were analyzed, patients who had surgery from 1998 to 2003, and those who had surgery from 2004 to 2008. Stress UI rates after RP were determined in both groups at 1, 3, 6 and 12 months following surgery with patient pad day usage. Urethrovesical anastomosis strictures were also analyzed in each group and their resolution.

RESULTS: 15 patients were excluded from the initial 137 because CT3 stage was detected, remaining 122 prostatectomies with $<$ CT2c stage in the study. Average age was 67 years (50-74). Mean preoperative PSA was $8.1 \mathrm{ng} / \mathrm{ml}$ (4.5-25). Biopsy Gleason score was < 6 in 70\% (85/122) and 7-8 in 30\% $137 / 122)$.

In the period between 1998 and 2003, 49 patients underwentsurgery and results were: initially continent $28.5 \%$ (14/49), continent after one month: $6.1 \%(3 / 49)$, at 3 months: $12.2 \%(6 / 49)$, six months $8.1 \%(4 / 49)$ and at a year: $22.4 \%(11 / 49)$. 20.4\% (10/49) remain with mild incontinence and moderate/severe $2 \%(1 / 49)$. In this period $78 \%$ of the patients remain continent and $22 \%$ incontinent. In the period 2004-2008, 73 radical prostatectomies were performed with the following continence rates: initial continence $44 \%$ (32/73), after one month 0 patients, 3 months $6.8 \%$ (5/73), 6 months $12.3 \%$ (9/73), a year later $24.6 \%$ (18/73). Mild incontinence was observed in $8.2 \%(6 / 73)$ and mode- rate-severe in $4 \%(3 / 73) .88 \%$ of the patients achieved continence and $12 \%$ remain incontinent.

CONCLUSIONS: Urinary incontinence is a low frequency disorder in patients who undergo RP. Continence rates have been improving during the last years along with surgical technique and urologic expertise. Anti-incontinence devices after PR are rarely necessary and use is overrated.

Keywords: Prostate cancer. Prostatectomy. Urinary incontinence.

\section{INTRODUCCIÓN}

La prostatectomía radical es, hoy en día, uno de los principales tratamientos con intención curativa que se pueden ofrecer a un paciente diagnosticado de un cáncer de próstata organoconfinado y con una expectativa de vida mayor de 10 años (4). Otras opciones de tratamiento en este tipo de pacientes incluyen la radioterapia externa, la braquiterapia e incluso la "vigilancia activa". Cada una de estas alternativas terapeúticas se asocia con diferentes efectos secundarios que pueden afectar de forma precoz y/o tardía a estos pacientes y condicionar su calidad de vida.

La técnica quirúrgica de la prostatectomía radical perineal fue inicialmente descrita por Young en 1905. Millin desarrolló la vía retropúbica en 1945 y Raboy publicó en 1997 el primer caso de prostatectomía radical laparoscópica por vía extraperitoneal (2). Estas vías de abordaje han ido evolucionando con el paso de los años y se han realizado indistintamente en cada hospital en función de la experiencia y/o preferencia del cirujano. Actualmente la vía perineal se realiza en muy pocos centros, siendo la prostatectomía radical retropúbica con conservación de las bandeletas neurovasculares (descrita por Walsh en 1982), la técnica más extendida, hasta el desarrollo en los últimos años de la vía laparoscópica y de la cirugía robótica.

Los objetivos fundamentales que se deben conseguir tras la cirugía radical de la próstata son un buen control del cáncer y/o la resección completa del tumor, la preservación postoperatoria de la continencia urinaria y el mantenimiento de la potencia sexual $(1,5)$.

Gracias a los avances en el diagnóstico precoz de este tipo de neoplasia, como la Ecografía transrectal y otras técnicas de imagen, la cinética del PSA y sus distintas isoformas y los nuevos esquemas 
de biopsia prostática, la mayoría de los pacientes que diagnosticamos hoy en día presentan un cáncer de próstata limitado a la glándula. La población de pacientes que vamos a tratar con más frecuencia son aquellos con PSAs bajos, Gleason $<6$ y tacto rectal normal (T1C) (6). Teniendo en cuenta que la mayoría serán pacientes jóvenes y que el resultado oncológico va a ser el adecuado, los resultados funcionales como la continencia y la potencia sexual se han convertido en una prioridad fundamental en este grupo de pacientes y en una obligación y en un reto para el urólogo. Aunque la mayoría de ellos consideran que su vida sexual e incluso la continencia urinaria pasan a un segundo plano sobre la opción de curar completamente el cáncer de próstata, éstos reclaman cada vez más que debemos intentar mantener su calidad de vida tras la prostatectomía (7).

Los resultados de la prostatectomía radical suelen presentarse por separado. El control oncológico de la enfermedad se suele expresar mediante la presencia o no de progresión bioquímica (PSA > $0,4 \mathrm{ng} / \mathrm{ml}$ tras la cirugía) o mediante la incidencia de márgenes quirúrgicos positivos. La continencia urinaria se evalúa mediante el número de compresas utilizadas al día (24 horas-Pad test, cuestionario validado por la ICS) y la potencia sexual mediante el cuestionario IIEF o ante la posibilidad de conseguir una erección que permita la penetración con o sin fármacos vasoactivos (IPDE-5). Para intentar objetivar y hacer comparables las diferentes series publicadas se han desarrollado sistemas de puntuación como el descrito por Salomon o la introducción de nomogramas con el objetivo de evaluar de forma combinada los resultados oncológicos y funcionales tras la prostatectomía radical (PR) (8). Pero cuando se analizan aisladamente los resultados funcionales, es la incontinencia urinaria el principal problema que condiciona y afecta de manera fundamental la calidad de vida de los pacientes, y aquel efecto secundario que preferirían resolver tras la cirugía, predominando significativamente sobre la potencia sexual.

Realizando una revisión sistemática de los resultados funcionales publicados podemos observar que el porcentaje de IU tras PR es muy variable según las distintas series. El margen es muy amplio y puede oscilar entre el $2,5 \%$ y el $87 \%$, según Foote. Estos datos se publicaron en 1991 y como veremos más adelante, se han mejorado de forma considerable en lo últimos años tras el desarrollo y evolución de la técnica quirúrgica de la prostatectomía.

Recientemente asistimos a la introducción cada vez más frecuente de dispositivos quirúrgicos, más o menos complejos, para solucionar la IU post PR. Desde la inyección de agentes inertes en la unión uretrovesical, a la colocación de mallas o cabestrillos bulbouretrales y hasta la colocación del esfínter artificial. Cada técnica tiene su indicación en función de la severidad de la IU, como se verá a lo largo del monográfico, aunque debido al porcetanje real de IU post $\mathrm{PR}$, el número de dispositivos que realmente son necesarios esté probablemente sobredimensionado.

El objetivo de este trabajo fue analizar los resultados funcionales en cuanto a la incontinencia urinaria (IU) tras PR que tenemos en nuestro centro en los últimos diez años y realizar una revisión de la literatura sobre la evolución de la técnica quirúrgica y con ello de la reducción del porcentaje de IU tras PR.

\section{Definición IU postprostatectomía}

La incidencia de IU post PR es muy variable según las diferentes series publicadas. Estas diferencias se deben en parte a la manera de caracterizar la IU, ya que el porcentaje de IU varía sensiblemente en función de la valoración del cirujano, del paciente, en base a cuestionarios de calidad de vida y/o a estrictos criterios urodinámicos (3). El uso de cuestionarios validados realizados por los pacientes ha permitido mejorar la estandarización de la definición de la IU post PR, eliminar el sesgo del propio cirujano y facilitar la comparación entre diversos centros $(9,10)$. Pero estos cuestionarios tienen el inconveniente de que la pérdida urinaria que reflejan los pacientes no necesariamente coincide con su pérdida urinaria real, por lo que se hacen precisas otras herramientas más objetivas como el Pad test, que cuantifica el $n^{\circ}$ de compresas que precisa el paciente en un periodo de tiempo $(9,11)$.

Un factor importante es el tiempo de seguimiento tras la cirugía, es decir, desde cuando estamos considerando incontinente al paciente. Se necesita al menos un año después de la PR para poder definir el estado funcional del mismo, ya que la IU post PR suele ser pasajera y se produce una mejoría evidente en las tasas de continencia conforme avanzan los meses tras la intervención $(8,9,12,13)$.

De hecho, se aconseja esperar al menos un año tras la cirugía para plantearse la colocación de algún dispositivo anti-incontinencia.

Otros factores que influyen en que los resultados publicados sean tan dispares son la técnica quirúrgica empleada (conservación o no de las bandeletas neurovasculares, preservación del cuello vesical, etc) y la presencia de IU en el paciente previamente a la cirugía. De esta manera, se producen importantes 
sesgos en los estudios realizados al no ser los grupos homogéneos siendo difícil definir con exactitud la IU post PR.

Por tanto, para definir a un paciente como incontinente tras la realización de la PR es preciso, primero utilizar una herramienta de medición objetiva como el Pad test, y segundo realizar la valoración como mínimo al año de la intervención quirúrgica.

También es importante diferenciar cuál es la causa de la IU post PR, el déficit esfinteriano intrínseco, la hiperactividad del detrusor o una combinación de ambas. El déficit esfinteriano intrínseco se puede definir como la afectación del esfínter externo que plantea una presión de fuga $<$ de $20-30 \mathrm{~cm}$ de agua y con ello IU de esfuerzo. Es un concepto, no sólo funcional, sino que tiene un sustrato de lesión anatómica del esfínter y un sustrato urodinámico en su demostración.

La gran mayoría de los autores consultados defienden que la deficiencia esfinteriana es la causa predominante y fundamental de la IU que se produce tras la cirugía radical de la próstata $(8,9,14)$.

\section{Factores preoperatorios que pueden influir en la IU post PR}

Se han descrito diferentes factores preoperatorios que pueden influir en la continencia de los pacientes sometidos a una prostatectomía radical.

La edad constituye un factor fundamental a la hora de disminuir el riesgo de IU post PR. Se considera que aquellos pacientes más jóvenes $k 65$ años), tienen más garantías de continencia tras la cirugía. Según Steiner la tendencia es clara en este aspecto, aunque él no encuentra diferencias estadísticamente significativas. Otros autores como Kundu y Catalona describen tasas de continencia del $95 \%$ en pacientes < 50 años y del $93 \%$ en aquellos < de 69 años.

La presencia de IU previa también se debería tener en cuenta al analizar la frecuencia de la IU postquirúrgica.

Factores relativos al propio tumor son también importantes. Estadios avanzados (cT3) y Gleason $>7$ se correlacionan con mayores tasas de incontinencia, probablemente porque en estos casos la infiltración del tumor sobre el cuello, ápex o lateralmente hacen técnicamente más difícil respetar las estructuras anatómicas implicadas en la continencia y la potencia $(8,13,22)$.
Otros factores como el volumen prostático o la presencia de RTU de próstata previa no se correlacionan de forma significativa con mayores tasas de incontinencia postquirúrgicas $(21,28)$.

\section{Factores de la técnica quirúrgica para mejo- rar la IU post PR}

Existen varios factores en la realización de la técnica quirúrgica de la prostatectomía radical que pueden mejorar los resultados funcionales y las tasas de continencia postquirúrgicas. Entre ellos están:

- Vía anterógrada versus vía retrógrada: La realización de la prostatectomía retropúbica por vía retrógrada fue descrita por Walsh en 1983. Esta técnica permite una exposición óptima de la próstata, de su relación con el cuello vesical y de las bandeletas neurovasculares. La vía anterógrada fue descrita por Campbell en 1959 y permite una mejor exposición del ápex prostático y del esfínter estriado uretral. Los resultados oncológicos y funcionales son similares y la decisión dependerá de la experiencia del cirujano.

- Disección del ápex prostático y del esfínter estriado de la uretra: La disección del ápex y el corte de la uretra en su porción anterior se presentan como una de las partes más complejas de la PR por su situación retropúbica y supone una maniobra fundamental para conseguir buenas tasas de continencia postoperatorias por la posibilidad de lesionar el esfínter estriado uretral. A este nivel, también es frecuente lesionar las bandeletas neurovasculares que discurren laterales a la uretra aumentando la frecuencia de incontinencia e impotencia (21). En este sentido, la vía anterógrada para realizar la prostatectomía descrita por Campbell en 1959, favorece la movilización del ápex, disminuye la probabilidad de lesionar el esfínter estriado y permite obtener una longitud uretral adecuada para realizar una anastomosis con garantías en cuanto a la continencia (24).

- Conservación de las bandeletas neurovasculares: Las bandeletas neurovasculares se componen de elementos vasculares como ramas de la arteria vesical inferior, de la arteria prostática y arterias y venas capsulares, y de elementos nerviosos como las ramas cavernosas del nervio pélvico. Las bandeletas discurren posterolateralmente a la próstata y emiten ramas vasculonerviosas que penetran en la cápsula prostática. También discurren laterales a la uretra y finalmente se dirigen a los cuerpos cavernosos (21). Para el urólogo es muy importante conocer la distribución y localización de las bandeletas, ya que durante la cirugía es sencillo lesionarlas y de esta forma el mantenimiento de la continencia y la potencia postquirúrgicas es más 
complejo. Durante la cirugía es frecuente la lesión de los haces neurovasculares durante la disección de los pedículos laterales de la próstata y su unión craneal con las vesículas seminales, así como durante la disección del ápex prostático (21).

Muchos autores están de acuerdo en que la conservación uni o bilateralmente de las bandeletas mejora sensiblemente las tasas de potencia postoperatorias. Por otro lado, se han publicado resultados que afirman que el mantenimiento de estas estructuras anatómicas pueden mejorar también las tasas de continencia postoperatorias (23).

Un tema controvertido al respecto es el hecho de que el intento de conseguir unos buenos resultados funcionales conservando las bandeletas, puede condicionar el resultado oncológico al aumentar el \% de márgenes positivos tras la cirugía (26).

\section{- Preservación del cuello vesical y tubulización ure-} tral: La preservación del cuello vesical en la prostatectomía radical es una técnica muy utilizada en los últimos años desde que fue introducida por Walsh en 1982 y supone un paso fundamental en la cirugía con el fin de conseguir buenos resultados en la continencia urinaria. La preservación del cuello vesical permite una anastomosis entre la mucosa vesical y la mucosa uretral más directa y estanca, reduciendo el riesgo de extravasado y de estenosis posquirúrgica (9). Se han publicado diversos estudios sobre la conveniencia de conservar o no el cuello vesical así como el valor funcional que puede ofrecer la realización de un tipo u otro de anastomosis uretrovesical, como la reconstrucción "en raqueta" del cuello, la preservación de los ligamentos puboprostáticos junto con la preservación cervical y/o la tubulización uretral, entre otras (15).

Las tasas de continencia son elevadas en series de Catalona, Walsh o Shelfo, con un $92 \%, 93 \%$ y $88 \%$ respectivamente, cuando se realiza la preservación del cuello de la vejiga. Un factor importante a tener en cuenta cuando se preserva el cuello vesical es, si de esta manera aumenta el porcentaje de márgenes quirúrgicos positivos, cuestión que se discutirá más adelante. Por ejemplo, Soloway publica un $1 \%$ de márgenes positivos en el cuello vesical cuando se realiza la preservación del mismo (37).

\section{Estenosis de la Unión Uretrovesical}

La frecuencia de la estenosis postquirúrgica de la unión uretrovesical tras la prostatectomia radical es muy variable según las distintas series y oscila entre el $1 \%$ (Shelfo) hasta el $17 \%$, según Noh. Las causas de la estenosis son variadas, como los diferentes materiales de sutura empleados, la presencia de hematomas en el cuello vesical, la extravasación urinaria excesiva o la técnica quirúrgica empleada para la realización de la anastomosis uretrovesical. Al parecer, la reconstrucción del cuello vesical "en raqueta" presenta un porcentaje mayor de estenosis según Noh, aunque otros autores como Poon o Lowe publican menores tasas de estenosis con este procedimiento, $11 \%$ y $8,2 \%$, respectivamente. La preservación del cuello vesical además de facilitar la recuperación precoz de la continencia urinaria, también disminuye la frecuencia de estenosis en la anastomosis, el 1 \% según Shelfo y Soloway o el 5 \% publicado por Poon.

\section{MATERIAL Y MÉTODOS}

Hemos realizado un estudio retrospectivo de los 137 pacientes sometidos a prostatectomía radical (retropúbica y laparoscópica) en el Hospital General Universitario de Elche, en el período comprendido entre 1998 y 2008 . Se analizaron dos grupos de pacientes, los intervenidos de 19982003 y aquellos que se operaron del 2004-2008, ambos inclusive. No se realizó distinción en cuanto a la vía de abordaje realizada en cada grupo. En ambos grupos se determinó el porcentaje de IU de esfuerzo tras $P R$, mediante el número de compresas utilizadas al día por cada paciente, al mes, 3 meses, 6 meses y al año de la cirugía. También se analizaron el número de estenosis de la anastomosis uretrovesical en cada grupo y cómo se resolvieron.

No se analizaron los resultados oncológicos ni la potencia sexual tras la PR en cada grupo. Los grupos fueron comparables y homogéneos en cuanto al tacto rectal, nivel de PSA y Gleason en la biopsia prostática, con estadios clínicos < cT2c. Se excluyeron a aquellos pacientes cT3.

Dentro de cada período de tiempo se definieron los siguientes grupos: Secos de inicio (tras la retirada de la sonda), continentes al mes, a los 3 meses, a los 6 meses, continentes al año e incontinentes. Dentro de los incontinentes se diferenciaron aquellos con IU leve (1-2 compresas/día) y con IU moderedasevera (> 2 compresas/día).

También se realizó una búsqueda bibliográfica mediante el sistema PubMed para analizar la incidencia y los factores pre-postquirúrgicos implicados en la IU post PR en las diferentes series publicadas. 


\section{RESULTADOS}

De los 137 pacientes iniciales, se excluyeron 15 de ellos por tratarse de estadios cT3, resultando el total en 122 prostatectomías con estadio < cT2c.

La edad media de los pacientes fue de 67 años (50-74). La media del PSA preoperatorio fue de $8,1 \mathrm{ng} / \mathrm{ml}(4,5-25)$. El Gleason en la biopsia fue $<$ de 6 en el $70 \%(85 / 122)$ y de $7-8$ en el $30 \%$ (37/122).

En el periodo comprendido entre 19982003 se intervinieron 49 pacientes y se obtuvieron los siguientes resultados: Continentes de inicio: 28,5 $\%(14 / 49)$, continentes al mes: $6,1 \%(3 / 49)$, continentes a los 3 meses: 12,2 \% (6/49), continentes a los 6 meses: 8, $1 \%$ (4/49), continentes al año: 22,4 $\%(11 / 49)$. Quedaron con IU leve el 20,4 \% (10/49) y con IU moderada/severa el $2 \%$ (1/49). En este periodo el $78 \%$ de los pacientes resultaron continentes $y$ el $22 \%$ incontinentes.

En el periodo de 2004-2008 se realizaron 73 prostatectomías radicales con las siguientes tasas de continencia: Continentes de inicio: $44 \%$ (32/73), continentes al mes: 0 pacientes, continentes a los 3 meses: 6,8\% (5/73), continentes a los 6 meses: $12,3 \%(9 / 73)$, continentes al año: $24,6 \%$ (18/73). Quedaron con IU leve el $8,2 \%(6 / 73)$ y con IU moderada/severa el $4 \%$ (3/73). Resultaron continentes un total del $88 \%$ de los pacientes e incontinentes el $12 \%$.

Desde 1998 hasta 2008 encontramos un 9 $\%(11 / 122)$ de estenosis de la unión uretrovesical, que se resolvieron con dilataciones/sondaje en 6 casos y con uretrotomía interna endoscópica/apertura con láser Holmiun en 5 de ellos (45\%). De los 11 casos, 4 de ellos presentan una IU leve con los es- fuerzos y necesitan 1 comp/día. El resto están secos y con un hábito miccional normal.

Los datos de continencia en ambos periodos quedan recogidos en la Tabla I.

\section{DISCUSIÓN}

La prostatectomía radical es el tratamiento de elección en los pacientes con cáncer de próstata organoconfinado y con una expectativa de vida mayor de 10 años (15). Actualmente en la gran mayoría de los centros la PR se realiza por vía abierta (retropúbica), por vía laparoscópica (intra y/o extraperitoneal) o mediante la cirugía robótica. La técnica de la prostatectomía radical retropúbica es la descrita por Walsh en 1982 y consta de la preservación de las bandeletas neurovasculares y del cuello vesical, permite un control óptimo del complejo venoso dorsal y la disección completa y cuidadosa del ápex prostático facilitando una anastomosis uretrovesical precisa, segura y estanca. Con esta técnica es posible conseguir buenos resultados oncológicos y funcionales reduciéndose de forma significativa las tasas de incontinencia e impotencia postquirúrgicas.

Actualmente los pacientes que se diagnostican de cáncer de próstata son cada vez más jóvenes y generalmente el tumor estará organoconfinado a la glándula. En este punto, garantizado un buen control oncológico de la enfermedad, es imprescindible conseguir un buen resultado funcional en cuanto a la continencia y potencia postoperatorias. De hecho, los propios pacientes se decantarán por uno $\mathrm{u}$ otro tratamiento de los que le ofertemos en función de lo que éste afecte a su calidad de vida, siendo fundamental para el urológo intentar preservar el estado funcional del paciente tras la cirugía.

\section{TABLA I. CONTINENCIA EN LOS DOS PERIODOS DE REVISIÓN.}

\begin{tabular}{|c|c|c|c|c|c|c|c|c|c|c|}
\hline & № & Inicio & $\mathbf{1} \mathbf{~ m}$ & $\mathbf{3} \mathbf{m}$ & $\mathbf{6} \mathbf{m}$ & Año & IU L & IU MoSe & Cont & Inc \\
\hline $1998-2003$ & 49 & $28,5 \%$ & 6,1 & $12 \%$ & $8,1 \%$ & $22,4 \%$ & $20,4 \%$ & $2 \%$ & $78 \%$ & $22 \%$ \\
\hline $2004-2008$ & 73 & $44 \%$ & - & $6,8 \%$ & $12,3 \%$ & $24,6 \%$ & $8,2 \%$ & $4,1 \%$ & $88 \%$ & $12 \%$ \\
\hline
\end{tabular}

*IU L: Incontinencia urinaria leve. // *IU MoSe: Incontinencia urinaria moderada-severa. 
Como ya se hemos comentado, para definir a un paciente como incontinente tras la realización de la PR es preciso, primero utilizar una herramienta de medición objetiva como el Pad test, y segundo realizar la valoración como mínimo al año de la intervención quirúrgica.

Hay autores como Litwin que afirman que aunque el control oncológico al año de la prostatectomía puede ser adecuado (85\%), los resultados funcionales pueden no ser comparables, con tasas de continencia del $65 \%$, sensiblemente inferiores a otras series publicadas. Las diferencias en cuanto al porcentaje de IU postPR es muy variable entre los diferentes autores. Según Foote la IU postPR puede oscilar entre el $2,5 \%$ y el $87 \%$, aunque estos son datos de 1991. Catalona publica en 1993 un $94 \%$ de continentes totales al año de la cirugía, sobre más de 400 prostatectomías. En series más recientes, Walsh obtiene un $93 \%$ de continentes realizando la vía retrógrada y Kundu hasta un $96 \%$ en pacientes menores de 60 años, sobre un total de más de 2700 procedimientos. Curto y Stolzenburg obtienen tasas de continencia del $95 \%$ y $92 \%$, respectivamente con la vía anterógrada laparoscópica. Por otra parte, Menon publica en 2007 unas tasas de continencia del $94 \%$ con cirugía robótica, muy similares al resto de las series.

Muchos de estos resultados provienen de hospitales de referencia de talla internacional, siendo difícil su comparación con resultados de hospitales más pequeños y con menor número de procedimientos realizados. Aún así, se puede comprobar con lo revisado en la literatura que en centros menores también se consiguen buenos resultados funcionales, con un $80 \%$ de continencia según Gaylis en 1998 o un 93 \% según Cohn en 2002 sobre un total de 398 prostatetomías.

Nuestros resultados advierten que se intervinieron menos pacientes en el primer periodo de tiempo debido a que en nuestro centro en los años iniciales, se trataban más casos con radioterapia externa, mientras que en años más recientes la prostatectomía radical retropúbica y laparoscópica se han convertido en el tratamiento de elección para estos pacientes. También cambiamos el esquema de biopsia prostática a un esquema de 10-12 cilindros en primeras biopsias aumentando significativamente la tasa diagnóstica y con ello el número de pacientes candidatos a prostatectomía. Se puede observar que la tasa de continencia ha mejorado en un $10 \%$ en estos 10 años de estudio, del $78 \%$ hemos pasado al $88 \%$ de pacientes continentes totales al año de la intervención. Estos resultados son ligeramente inferiores a otras series publicadas, probablemente porque en los últimos 3 años hemos estado en la fase de la curva de aprendizaje en prostatectomía radical laparoscópica y las cirugías han sido técnicamente más dificultosas y con peores resultados iniciales.

Un factor importante que puede condicionar el buen resultado funcional tras la cirugía es la selección previa del paciente en cuanto a la edad, presencia de IU previa, técnica quirúrgica empleada, etc (1). Muchos autores defienden que cuando la PR se realiza en pacientes jóvenes (< 65 años) se consiguen mejores tasas de continencia. Kundu y Catalona describen tasas de continencia del 95 $\%$ en pacientes < 50 años y del $93 \%$ en aquellos < de 69 años, sobre una serie de más de 2700 prostatecomías realizadas durante 10 años y con un seguimiento de más de 18 meses tras la cirugía. Stanford también considera la edad un factor predictor significativo para conseguir buenos resultados funcionales, de forma que obtiene un $91 \%$ de continentes totales en pacientes < de 60 años frente al $84 \%$ del grupo de edad comprendido entre 65 74 años.

Otros factores importantes son los relativos a la técnica quirúrgica propiamente dicha, como la elección de la vía anterógrada o retrógrada, preservación el cuello vesical y/o la conservación de las bandeletas neurovasculares. En último término, la vía y la técnica utilizada dependerán de la experiencia del urólogo y será aquella con la que éste se sienta más cómodo. Los resultados de la vía anterógrada o retrógrada son similares en cuanto a control oncológico y funcional. Hay autores como Walsh, Kundu o Stanford que se decantan por la vía retrógrada con tasas de continencia del 91-93 $\%$. Otros autores como Carini prefieren la vía retrógrada con resultados de continencia similares.

En general, cuando se realiza la preservación del cuello de la vejiga las tasas de continencia son elevadas en series de Catalona, Walsh o Shelfo, con un $92 \%$, $93 \%$ y $88 \%$ respectivamente. También es importante el tipo de reconstrucción del cuello vesical que se realiza. Noh defiende que la preservación del cuello vesical junto con la sección de los ligamentos puboprostáticos cercana a la próstata ofrece similares tasas de continencia frente a la reconstrucción "en raqueta" del cuello, aunque el porcentaje de estenosis de la anastomosis son mayores con este último método.

Hay autores que sostienen que la preservación del cuello vesical puede suponer el aumento del porcentaje de márgenes quirúrgicos positivos. Soloway publica un $1 \%$ de márgenes positivos en 
el cuello vesical, sobre una serie de más de 600 prostatectomías con preservación del mismo. Shelfo describe tan sólo un 0,5\% de márgenes positivos tras la preservación del cuello vesical y la realización de 265 procedimientos. Otros autores como Braslis obtienen márgenes positivos en el cuello de hasta el $36 \%$, aunque esto es más frecuente cuando se intervienen pacientes en estadios localmente avanzados (T3). Autores como Razi no encuentran diferencias estadísticamente significativas en cuanto al porcentaje de márgenes cervicales positivos entre la preservación del cuello y la reconstrucción del mismo. Noh publica un $9 \%$ de márgenes positivos en el cuello con independencia de si se realiza preservación cervical versus reconstrucción en raqueta.

Es conocido por todos que durante la prostatectomía radical es frecuente la lesión de los haces neurovasculares durante la disección de los pedículos laterales de la próstata y su unión craneal con las vesículas seminales, así como durante la disección del ápex prostático (21). La conservación uni o bilateralmente de las bandeletas mejora sensiblemente las tasas de potencia postoperatorias, según muchos autores. Por otro lado, se han publicado resultados que afirman que el mantenimiento de estas estructuras anatómicas puede mejorar también las tasas de continencia postoperatorias. Bukhard afirma que no hay diferencias estadísticamente significativas en cuanto a la continencia postoperatoria entre la conservación de una o de las dos bandeletas, si bien sí encuentra diferencias a favor de la conservación de los haces neurovasculares frente a extraerlos con la próstata. En su estudio sostiene que existe hasta el $14 \%$ de diferencia en la tasa final de continencia a favor de la conservación. Otros autores como Selli y Smither también defienden la conservación de las bandeletas con el fin de mejorar la continencia urinaria, consiguiendo tasas de continencia cercanas al $91 \%$.

Un tema controvertido al respecto es el hecho de que el intento de conseguir un bueno resultado funcional conservando las bandeletas, puede condicionar el resultado oncológico al aumentar el $\%$ de márgenes positivos tras la cirugía, aunque esto es más frecuente en tumores más avanzados que en aquellos organoconfinados. $(9,21)$

La estenosis de la unión uretrovesical es una complicación postoperatoria que hay que tener en cuenta porque puede condicionar problemas en el hábito miccional e incluso incontinencia urinaria, aunque suele ser una incontinencia de urgencia por hiperactividad del detrusor secundaria a la obstrucción infravesical.
Selli publica un 6,8 \% de estenosis de la anastomosis sobre un total de 131 prostatectomías con preservación del cuello vesical, necesitando la apertura endoscópica de la estenosis en el $88 \%$. Kundu, sobre una revisión de más de 3400 prostatectomías, describe un porcentaje de estenosis de la anastomosis del 2,7\%, muy reducido desde la recontrucción del cuello vesical sobre una sonda de $22 \mathrm{Ch}$. Cuando realizaban la reconstrucción sobre un calibre de sonda menor (18 Ch), el porcentaje de estenosis fue del $8 \%$. Según Noh el porcentaje de estenosis cuando se realiza una reconstrucción del cuello "en raqueta" ascendió al 17,7\% frente al $5,2 \%$ tras la preservación cervical convencional. Otros autores como Poon también encuentran diferencias similares, $11 \%$ con la técnica "en raqueta" frente al $5 \%$. En nuestra serie el porcentaje del $9 \%$ estenosis de la unión es semejante a las otras series publicadas y más de la mitad de ellas se resolvieron con medidas conservadoras como las dilaciones periódicas o el sondaje vesical.

Finalmente cuando se analizan estudios de calidad de vida, podemos observar que la satisfacción de los pacientes tras la prostatectomía radical es elevada y que muchos de ellos volverían a escoger este tratamiento si fuera necesario. Estudios de Jonler y Braslis demuestran una satisfacción tras la cirugía del $88 \%$ y del $92 \%$, respectivamente.

Llegados a este punto es importante discutir si son realmente necesarios los dispositivos antiincontinencia para los pacientes incontinentes tras la PR. Kundu y Catalona describen únicamente la necesidad de colocar un esfínter artificial por IU severa tras PR en el 0,3\% de los más de 2700 pacientes revisados en su serie. En este sentido se pronuncian la gran mayoría de los trabajos publicados. Esto último y nuestra propia experiencia como cirujanos nos hacen ver que el porcentaje real de IU postPR es bajo. Además la gravedad de la IU de aquellos que quedan incontinentes es mayoritariamente poco importante, quedando limitada a una IU de esfuerzo leve, que precisa menos de 1-2 compresas al día y que limita escasamente la calidad de vida de los pacientes. Por otro lado, la colocación de estos dispositivos no está exenta de complicaciones y supone la realización de otra intervención quirúrgica a lo que muchos pacientes no están dispuestos.

En cualquier caso lo que se desprende de todas las series consultadas es que con el paso de los años y con la mejora en la técnica quirúrgica los pacientes que quedan incontinentes tras la prostatectomía radical son muy pocos y aquellos que van a precisar algún dispositivo antiincontinencia para resolver su problema son cada vez menores. 


\section{CONCLUSIONES}

La prostatectomía radical permite un control óptimo del cáncer de próstata organoconfinado con buenos resultados funcionales en cuanto a continencia postoperatoria en manos expertas. En la última década asistimos a una mejora evidente en las tasas de continencia tras la cirugía como consecuencia del refinamiento en la técnica quirúrgica. La vía laparoscópica o la novedosa cirugía robótica obtienen similares resultados funcionales a la prostatectomía retropúbica convencional.

Debido a que el porcentaje de pacientes que continúan incontinentes tras la cirugía es escaso y que la severidad de estas incontinencias no precisará tratamiento en su mayoría, la necesidad real de los dispositivos antiincontinencia como tratamiento de la IU postPR es escasa y su utilización cotidiana nos parece sobredimensionada.

\section{BIBLIOGRAFÍA Y LECTURAS

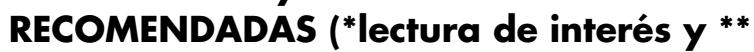 lectura fundamental)}

*1. Salomon L, Leurel O, Anastasiadis A, et al. Combined reporting of cancer control and functional results of radical prostatectomy. Eur Urol, 2003; 44: 656-60.

2. Salomon L, Saint F, Anastasiadis A, t al. Outcome and complications of radical prostatectomy en patients with PSA $<10 \mathrm{ng} / \mathrm{ml}$ : comparison between the retropubic, perineal and laparoscopic approach. Prost Canc and Prost Dis, 2002; 5:285-90.

3. Foote J, Yun S, Leach GE. Postprostatectomy incontinence. Pathophysiology, evaluation and management. Urol Clin North Am 1991; 18:229-41.

*4. Stanford J, Feng Z, Hamilton A, et al. Urinary and sexual function after radical prostatectomy for clinically localized prostate cancer. JAMA, 2000;283: 354-60.

5. Saranchuck J, Kattan M, Elkin E, Touijer K, Scardino P, Eastham J. Achieving optimal outcomes after radical prostatectomy. J Clin Oncol, 2005; 23: 4146-51.

6. Partin AW, Mangold LA, Lamm DM, Walsh P, Epstein J, Pearson JD. Contemporary update of prostate cancer staging nomograms (Partin tables) for the new millennium. Urology, 2001;58: 8438.

7. Fowler F, Barry M, Lu-Yao G, Wasson J, Roman A, Wennberg J. Effect of radical prostatectomy for prostate cancer o patient quality of life: results from a medicare survey. Urology, 1995;45: 100713.
*8. Smither A, Guralnick M, Davis N, See W. Quantifying the natural history of post-radical prostatectomy incontinence using objective pad test data. BMC Urology 2007; 7:2: 1-6.

9. Selli C, De Antoni P, Moro V, et al. Role of bladder neck preservation in urinary continence following radical retropubic prostatectomy. Scan J Urol Nephrol, 2004;38: 32-7.

10. Carlson K, Nitti V. Prevention and management of incontinence following radical prostatectomy. Urol Clin North Am 2001; 28: 592-612.

11. Donnelan S, Duncan H, MacGregor R, Russel J. Prospective assessment of incontinence after radical prostatectomy: objective and subjective analysis. Urology, 1997; 49: 225-30.

12. Lepor H, Kaci L, Xue X. Continence following radical retropubic prostatectomy using self-reporting instruments. J Urol, 2004; 171: 1212-15.

13. Eastham $\mathbf{J}$ et al. Risk factors for urinary incontinence after radical prostatectomy. J Urol, 1996; 156:1707-13.

14. Ficazzola M, Nitti V. The etiology of post-radical prostatectomy incontinence and correlation of symptoms with urodinamics findings. J Urol, 1998; 160(4): 1317-20.

*15. Noh C, Kshirsagar A, Mohler J. Outcomes after radical retropubic prostatectomy. Urology, 2003; 61(2): 412-16.

16. Poon M, Ruckle H, Bamshad B, Tsai C, Webster R, Lui P. Radical retropubic prostatectomy: bladder neck preservation versus reconstruction. $\mathrm{J}$ Urol, 2000; 163: 194-98.

17. Lowe B. Comparison of bladder neck preservation to bladder neck resection in maintaining postprostatatectomy urinary continence. Urology, 1996;48: 889-93.

18. Shelfo S, Obek C, Soloway M. Update of bladder neck preservation during radical retropubic prostatectomy: impact on pathologic outcome, anastomotic strictures and continence. Urology, 1998; 51:73-8.

*19. Soloway M, Neulander E. Bladder neck preservation during radical retropubic prostatectomy. Semi Urol Oncol 2000; 18: 51-6.

20. Walsh P, Donker P. Impotence following radical prostatectomy: insight into etiology and prevention. J Urol 1982; 128: 492-97.

*21. Steiner M. Current results and patient selection for nerve-sparing radical retropubic prostatectomy. Sem Urol Oncol 1995; 13(3): 204-14.

22. Egawa S, Minei S, Iwamura M, Uchida T, Koshiba K. Urinary continence following radical prostatectomy. Jpn J Clin Oncol 1997; 27: 71-5.

23. Buckhard F, Kessler T, Fleischmann A, et al. Nerve sparing open radical retropubic prostatectomydoes it have and impact on urinary continence? J Urol 2006;176: 189-95. 
*24. Carini M, Masieri L, Minervini A, Lapini A, Serni S. Oncological and functional results of anterograde retropubic prostatectomy for the treatment of clinically localised prostate cancer. Eur Urol 2008: 53:554-63.

25. Campbell EW. Total prostatectomy with preliminary ligation of the vascular pedicles. J Urol 1959; 81: 464-7.

26. Litwin M, MacGuigan K, Spahall A, Dhanani N. Recovery of health-related quality of life in the year after radical prostatectomy: early experience. J Urol 1999; 61: 515-9.

*27. Kundu S, Roehl K, Eggeneer S, Antenor J, Han $\mathrm{M}$, Catalona W. Potency, continence and complications in 3,477 consecutive radical retropubic prostatectomies. J Urol 2004;172:2227-31.

28. Catalona W, Basler J, Return of erections and urinary continence following nerve spraring radical retropubic prostatectomy. J Urol 1953; 150:9057.

29. Braslis K, Petsch M, Lim A, Civantos F, Soloway M. Bladder neck preservation following radical prostatectomy. Eur Urol 1995;28:202-8.
30. Curto F, Benijts J, Pasandoro A, et al. Nerve sparing laparoscopic radical prostatectomy: our technique. Eur Urol 2006;49:344-52.

31. Stolzenburg U, Rabenalt R, Do M, et al. Endoscopic extraperitoneal radical prostatectomy: Oncological and functional results after 700 procedures. J Urol 2005; 174:1271-5.

32. Menon M, Shrivastava A, Kaul S, et al. Vattikuti Institute prostatectomy: contemporany technique and analisys of results. Eur Urol 2007;51:648-58.

33. Gaylis F, Friedel W, Armas O. Radical retropubic prostatectomy outcomes at a community hospital. J Urol 1998;159:167-71.

34. Cohn J, El-Galley R. Radical prostatectomy in a commnunity practice. J Urol 2002;167:224-8.

35. Jonler M, Messing E, Rhodes P, Bruskewitz R. Sequelae of radical prostatectomy. BJU 1994;74:352-8.

36. Braslis K, Santa-Cruz C, Brickman A, Soloway M. Quality of life 12 months after radical prostatectomy. BJU 1995;75:48-53.

37. Razi A, Yahyazadeh SR, Sedighi Gilani MA, Kazemeyni SM. Bladder neck preservation during radical retropubic prostatectomy and postoperative urinary continence. J Urol, 2009;6(1):23-6. 